\title{
A Numerical Study on the Performance of a Magnesium- Based Automotive Cooling Fan with Bead Structure
}

\author{
K. H. Hur, B. A. Haider and C. H. Sohn ${ }^{\dagger}$ \\ School of Mechanical Engineering, Kyungpook National University, Daegu, 41566, Korea \\ †Corresponding Author Email: chsohn@knu.ac.kr
}

(Received December 4, 2019; accepted June 17, 2020)

\begin{abstract}
This paper presents the numerical analysis of three types of magnesium-based, axial-flow automotive cooling fans. The numerical modeling is conducted for geometrically modified fan designs with and without bead structure. The effect of geometric modifications of the fan blades on the fan performances ( $P-Q$ curve $)$, fan efficiency, and energy efficiency is investigated using unsteady Reynolds-Averaged Navier-Stokes (URANS) equations with the sliding mesh methodology. The baseline fan having no-beads is fabricated using 3D printing technology and tested to measure the flow velocity and volumetric flow rate which shows good agreement to the numerical results. Subsequently, fans with beads are further optimized to achieve a significant increase in fan performances. To investigate the fan vibrations, modal analysis is also carried out using magnesium-alloy AZ31 as the fan material. The modal analysis gives natural frequencies of all types of fans which are beyond the fan rotational frequency and seems satisfactory.
\end{abstract}

Keywords: Axial-flow cooling fan; Bead structure; CFD; Fan performance; Modal analysis; Passive control.

\section{INTRODUCTION}

In modern vehicles, the thermal management by the cooling system within the engine compartment consists of several stacked heat exchangers such as a radiator, lubricant coolers, and condensers (Srinivasa et al. 2014). To dissipate the heat energy carried by the turbocharger, lubricating oil, condenser, and engine coolant, a sophisticated cooling fan system is required to produce a sufficiently uniform and high flow rate through these heat exchangers (Henner et al. 2003). On the contrary, the design of modern vehicles emphasizes minimizing the vehicle size by enhancing the aesthetics than on providing adequate cooling flow (Gifford et al. 2006). So, the front-end styling of such vehicles limits the size of underhood geometries and houses the engine block closer to the cooling fan.

Since the automotive cooling fans are axial-flow machines because of the simplicity, compactness, low-cost, and ability to deliver uniform cooling air, they usually operate at off-design conditions. However, the new stringent requirements of noise and emissions, airflow, pressure, and efficiency of cooling flow, the axial flow fans can no longer comply with these requirements (Williams et al. 2011). Alternatively, radial-flow fans (Hofe and Thien 1985; Soldner et al. 2001) have been proposed to overcome the aforementioned problems. The pressure production of a radial-flow fan is extremely efficient than an axial fan because of the airflow through the well-defined guided passages. A radialflow fan also named as a centrifugal fan since of the augmentation of a considerable swirl due to centrifugal effects. A modified axial flow impeller which generates a radial component of flow, added to the spiral flow, to achieve higher flow rate and pressure is another type of fan namely mixed-flow or lean-type fan (Yu et al. 2011). Such fan types require special design considerations like aerodynamically formed inlet and scroll as fan casing but the underhood space in cars hardly permits such a design. However, radial flow and mixed flow fans are always the first options in heavy-duty vehicles (Williams et al. 2011).

The axial-flow fans have been widely used in automotive cooling and in-cabin air recirculation systems. The benefit of the use of axial-flow fans to augment the heat transfer is evident particularly in the automotive industry since of the requirement of relatively compact designs. The extensive use of axial-flow fans for fluid flow and heat transfer has appeared in the detailed investigation within the performance attributes of many design options (Wang et al . 2015; Tare et al. 2017; Gullberg and Tavernier 2014; Gullberg et al. 2011). 
Nowadays, the engine compartment in automotive vehicles is becoming more crowded because of the integration of additional components which may include HVAC, power steering system, particle filters, and automatic gear systems. The influence of these added components is significant on the cooling requirements along with their thermal efficiency which has a direct impact on the energy budget and comfort aspects of a vehicle. Consequently, an adequate cooling airflow through the heat exchangers has become an essential element of vehicle thermal management. Moreover, an improvement in the vehicle's overall performance including the cooling system performance is desired while keeping in view the constraints of axial compactness.

To increase the performance of axial-flow fans, researchers have developed and investigated various techniques. Active and passive flow control techniques are among them which are utilized to suppress the flow separation and improve the efficiency of axial-flow fans (Bianchi et al . 2013; Bianchi et al. 2014). The present research presents a novel type of passive flow device to increase cooling airflow rate and hence the performance and energy efficiency of the fan. For this purpose, a baseline fan is geometrically modified by adding beads on the suction and pressure sides of the fan. Moreover, the size and location of the bead on the suction side is varied systematically to further improve the volumetric flow. Also, fan vibration is analyzed by modal analysis. The baseline fan has no beads is named as a no-beads fan (NBF), the bead on the fan suction side is named as a reverse-beads fan (RBF) whereas the beads on the pressure side are called forward-beads fan (FBF).

The axial-flow cooling fans have been extensively studied due to their versatile industrial and engineering applications. Eck (1973) defined various fan geometric parameters and carried out a series of systematic analyses that enables to evaluate fan performance. Later, Wallis (1983) developed a combined theory for airflow through the fan and duct design. Bleier (1998) studied the concepts explained by Wallis (1983) and performed calculations to make the preset requirements in volumetric flow rate, static pressure, power, and efficiency.

The cooling fan is the critical component of the thermal management system which draws additional air through the radiator, either by natural convection or ram air forced through the radiator due to vehicle speed. Therefore, an accurate and reliable prediction of cooling airflows is required to understand and estimate the performance of the cooling fan under realistic vehicle operating conditions. The air distribution and flow patterns in the vehicle underhood are highly complicated and transient due to the intricate nature of the cooling fan flows. Consequently, a high-fidelity simulation is essential to capture the relevant physics associated with cooling airflow prediction.

In recent years, an increase in computing capability and growth in the simulation field has observed which makes CFD a primary tool to model and simulate complex geometries accurately. Simulating rotating machinery using CFD, several methodologies exist which include approximate models based on pressure jump (Van der Spuy, Von Backström, and Kröger 2010) and actuator disk model (Thiart and von Backström 1993; Meyer and Krger 2001; Bredell et al. 2006) approaches. The pressure-jump approach and actuator disk methods represent the operation of the fan using momentum source terms and require no physical modeling of the fan. A more accurate representation of the rotating machinery is to simulate using moving (multiple) reference frames (MRF) approach (Luo and Gosman 1994) which requires an actual three-dimensional model of the fan geometry. The MRF approach is a steady-state approximation of a time-dependent solution. The sliding-mesh method is a variant of MRF to analyze unsteady flows as a transient simulation. Many researchers have successfully applied the MRF or sliding mesh approach to simulate rotational systems (Sahili et al. 2013; Haider et al . 2017a; Adeeb et al . 2016; Babich et al .2017).

Finally, this study aims to numerically investigate geometrically modified axial-flow automotive cooling fans: (1) no-beads fan, (2) forward-beads fan, and (3) reverse-beads fan. High-fidelity modeling is carried out based on the sliding mesh technique to accurately compute the volumetric flow rate and energy efficiency. Furthermore, the P-Q curve is developed for each fan type and compared. The rotation of the fan is varied from $300 \mathrm{rpm}$ to $3000 \mathrm{rpm}$. The no-beads fan is tested to ascertain the CFD simulation results for the subsequently modified fan shapes.

\section{Methodology}

\subsection{Fan Designs}

The baseline fan and blade geometric parameters are shown in Fig. 1 and Fig. 2, respectively. The baseline fan is used for experimental studies. Fig. 3(a) shows the geometrically modified fan with beads attached in the suction side of the fan blade, namely reversebeads fan (RBF) and Fig. 3(b) presents the beads installed at the pressure side of the fan blade, namely forward-beads fan (FBF). Table 1 shows the geometric and operating characteristics of the baseline and modified fans.

The magnesium-based baseline fan is fabricated by a mold that is made by a Stratasys Vantage 3-D printer. This 3-D printer is based on fused deposition modeling (FDM) technology. Fig 4(a) shows the 3D printer and Fig. 4(b) presents the finished product. The surface of the fabricated fan is smooth enough with a roughness factor of fewer than three microns.

\subsection{Experimental Setup}

The experimental setup for testing the baseline axial flow fan is shown in Fig. 5. The testbed is made of $60 \times 40 \times 3 \mathrm{~cm}$ aluminum alloy. To eliminate the vibrations caused by the rotating fan, four supporting cushions are used around the circular duct. The circular duct size is $\varnothing 30 \times 30 \mathrm{~cm}$. The fan is installed 


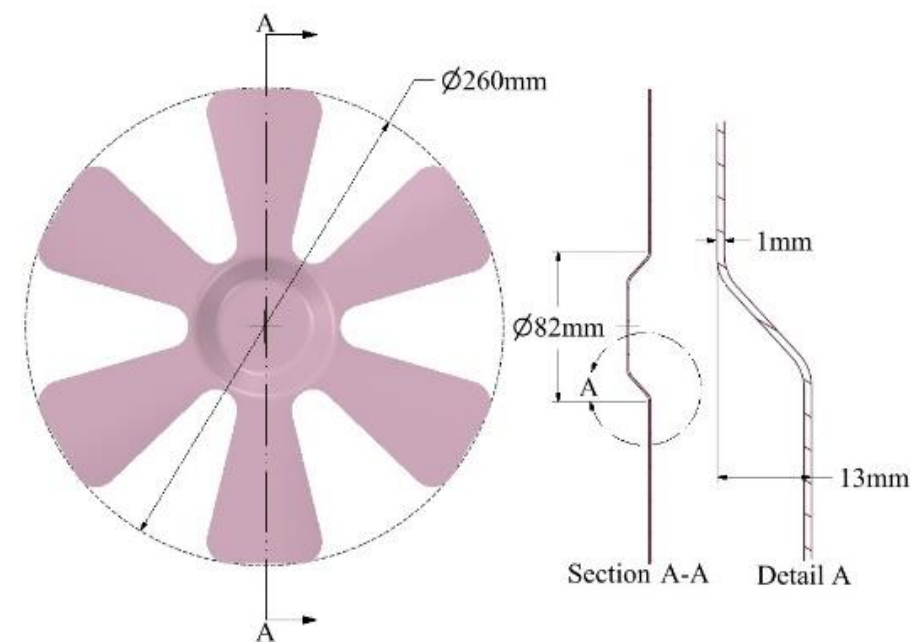

Fig. 1. Geometric representation of baseline fan (NBF). Illustrating the main dimensions of the baseline fan.

Table 1 Geometric and operating specifications of the cooling fan.

\begin{tabular}{|c|c|}
\hline Application & Automotive engine cooling \\
\hline Fan diameter & $260 \mathrm{~mm}$ \\
\hline Hub outer diameter & $82 \mathrm{~mm}$ \\
\hline Number of blades & 6 \\
\hline Blade thickness & $1 \mathrm{~mm}$ \\
\hline Operating range of speed & $300 \sim 3000 \mathrm{rpm}$ \\
\hline Rotation direction & CCW from the front-end \\
\hline
\end{tabular}

at one end of the duct whereas the other end is open to the atmosphere. A thermo-anemometer (LV111) is traversed at various measuring points in a circular section consists of four diameters and three measuring points per radius. The traverse points shown in Fig. 6 are sited on each dimeter according to the log-linear rule (Winternitz and Fischl 1957). The arithmetic mean of the individual velocity measurements at each traverse point is the mean flow velocity through the measuring plane. This mean flow velocity is then multiplied with the duct crosssectional area to obtain a volumetric flow rate.

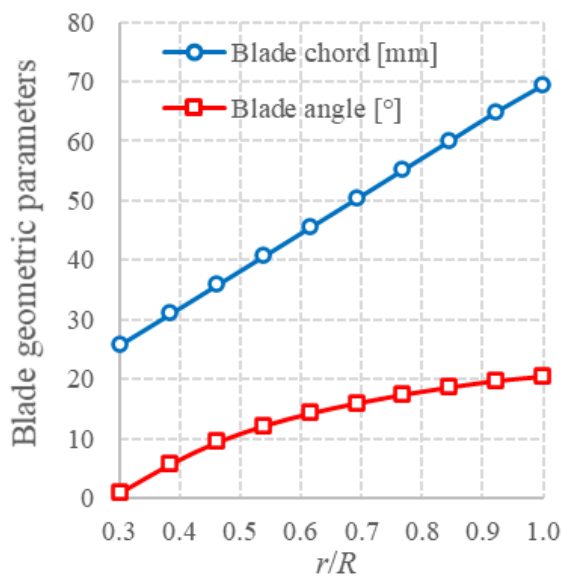

Fig. 2. Blade chord length and angle as a function of the normalized radial distance of the fan blade.

\subsection{Numerical Setup}

In the numerical part of the study, a commercially available finite volume-based solver, Ansys Fluent, is used. The simulations are performed by solving 3$\mathrm{D}$, incompressible, unsteady Reynolds-averaged Navier-Stokes (URANS) equations using secondorder finite volume discretization. A shear stress transport (SST $k-\omega)$ turbulence model is applied for all computations which exhibit an effective blend of accurate and robust near-wall formulation of the $k-\omega$ model with the freestream independence in the farfield region of the $k-\varepsilon$ model (Nobile et al. 2014; Chen et al. 2018). For advection terms and turbulence numeric set to second order in all cases.

In the computational domain, a single fan blade is modeled considering the rotational periodicity to save computational time. The computational domain for the analysis consists of a $60^{\circ}$ sector with three fluid zones in the following order, (i) inlet (static) fluid zone, (ii) rotating (sliding) fluid zone, and (iii) outlet (static) fluid zone. These static and rotating fluid zones are connected through a sliding interface to transfer the flow variables among each fluid zone. The inlet fluid zone is $2.5 \mathrm{D}$ upstream of fan inlet and outlet fluid zone is $5.0 \mathrm{D}$ downstream of fan outlet whereas all the fluid zones are extended $1.1 R$ in the radial direction.

The computational domain and boundary conditions applied to fan simulations are shown in Fig. 7. At the inlet, the mass flow rate is imposed whereas the outlet is set as a pressure outlet. The fan blade is set 

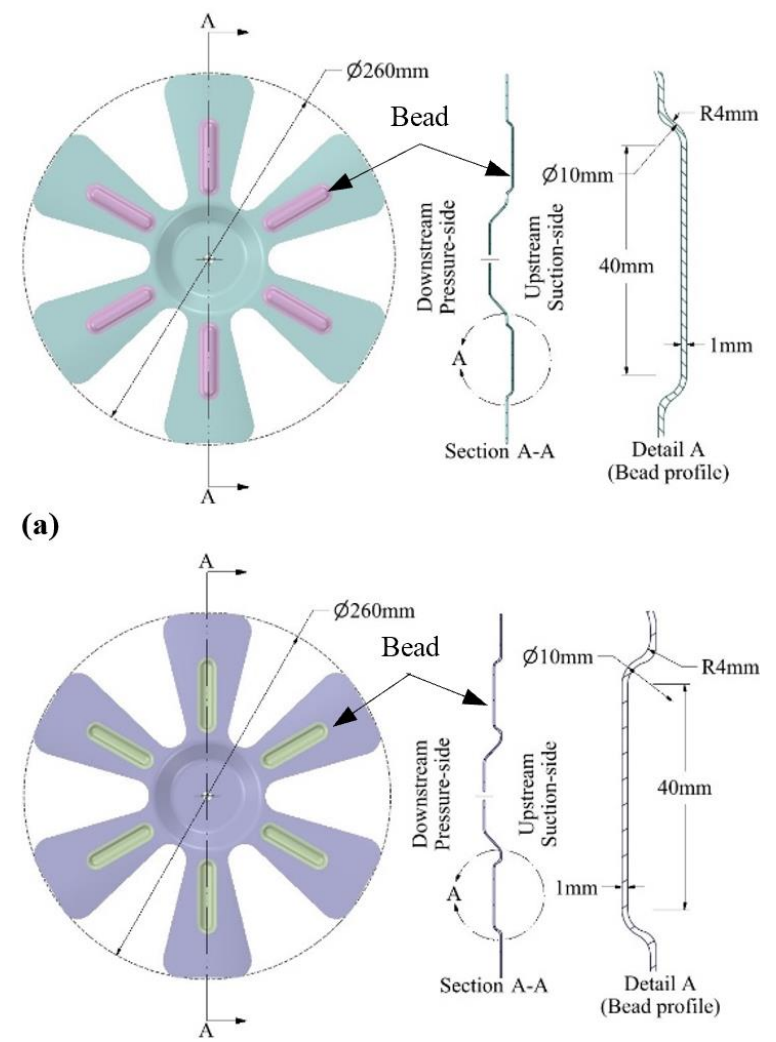

(b)

Fig. 3. Geometric illustration of (a) reverse-beads (RBF), and (b) forward-beads (FBF) fans.

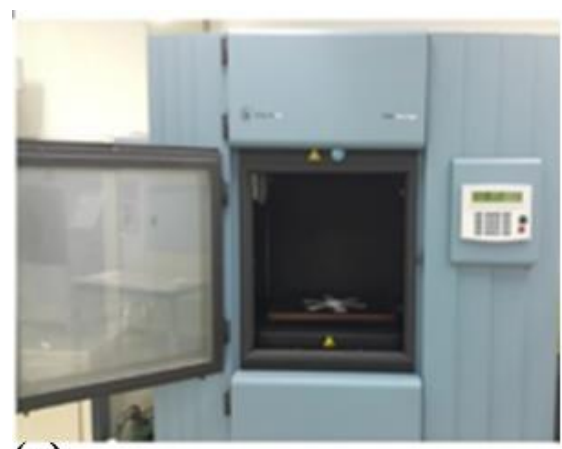

(a)

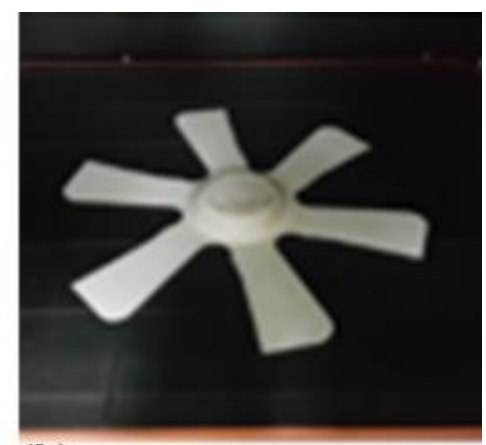

(b)

Fig. 4. (a) Stratasys Vantage 3-D printer, (b) a fan fabricated by a mold that is made with a 3-D printer.

as a rotational no-slip wall and adiabatic. Since the flow is periodic, only one blade is modeled, and the periodic condition is applied at the boundary. The duct walls are also set as no-slip walls. The timestep size is chosen such that the fan blade rotates $1^{\circ}$ in each timestep. A total of twenty fan blade rotations are simulated to get them periodically stable and converged solution.

The unstructured meshing provides flexibility in the mesh generation process and is widely used in complex rotating flow simulations (Haider et al . 2017b). Therefore, in this study, all the surfaces of the computational domain and fan blade have meshed with an unstructured grid of tetrahedrons. The fluid volume zones are filled with hexacore elements to save solution time by reducing the overall cell count. The mesh is refined near the fan blade surface by employing inflation layers to capture the boundary layer. The growth of the inflation layers over the blade surface is controlled according to Eq. (1).

$H_{p}=h \times g^{n-1}$

where $H_{p}$ is the height of the last prism, $h=0.01 \mathrm{~mm}$ is the height of the first layer, $g=1.2$ is the growth rate, and $n=15$ represents the number of inflation layers. The inflation layers provide an average wall $y^{+}$over the blade surface around one. Figure 8 shows the computational grid used in the study. 


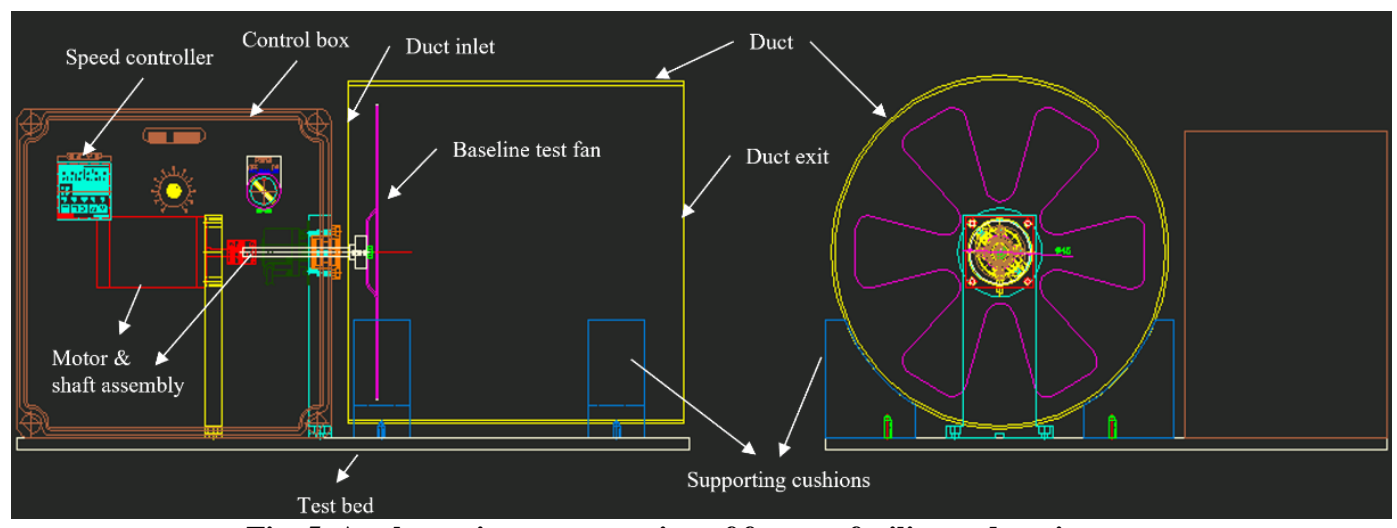

Fig. 5. A schematic representation of fan test facility and equipment.

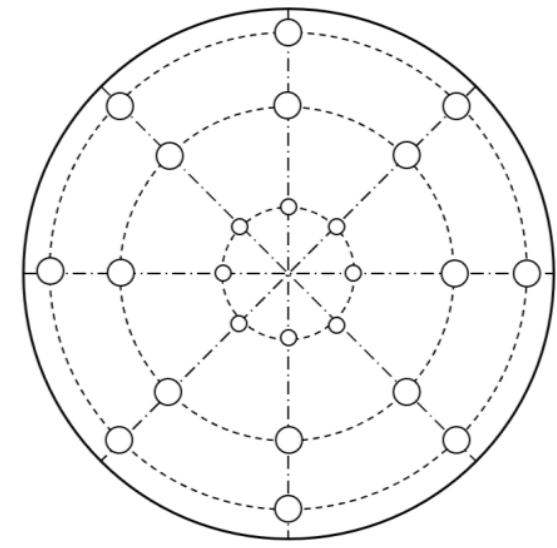

Fig. 6. Flow velocity measuring points in a circular duct according to log-linear rule (Winternitz and Fischl 1957).

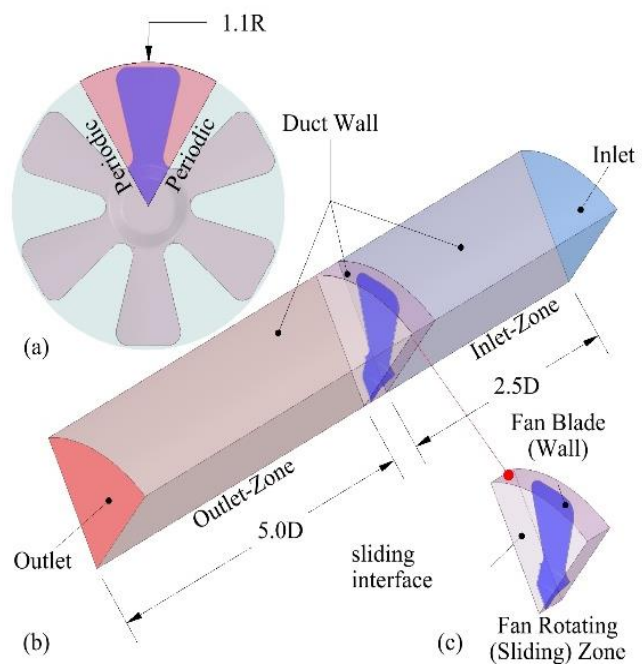

Fig. 7. Computational domain used in the automotive cooling fan simulations.

The grid sensitivity analysis is also carried out for the baseline fan to obtain the grid-independent solution. Three grids of sizes 0.6 (coarse), 1.2 (medium), and 2.4 (fine) million cells have been utilized in the grid sensitivity study. Table 2 shows the grid statistics and the resulting volumetric flow rate $(Q)$ in cubic- meters-per-second. The convergence of each case with coarse, medium, and fine grids is sufficiently ensured. Based on the grid sensitivity study, the numerical uncertainty in the volumetric flow rate for the baseline fan design is estimated at less than $1.0 \%$ with a grid convergence of second-order.

A complete CFD procedure starting from the objective till the documentation of the results passing through various essential processes like formulation of flow problem, geometry modeling, making highquality grids, solve the flow with appropriate solver settings, achieving convergence, and interpreting results are shown in Fig. 9.

Table 2 Grid sensitivity study of baseline fan at $1500 \mathrm{rpm}$.

\begin{tabular}{|c|c|}
\hline $\begin{array}{c}\text { G1 (Coarse), G2 (Medium), } \\
\text { G3 (Fine) }\end{array}$ & $0.6 \mathrm{M}, 1.2 \mathrm{M}, 2.4 \mathrm{M}$ \\
\hline Grid refinement ratio, $r$ & 2.0 \\
\hline Volumetric flow rate, & $0.078,0.072$, \\
$Q\left(\mathrm{~m}^{3} / \mathrm{s}\right)$ & 0.070 \\
\hline Convergence order & $\approx 2.0$ \\
\hline Grid convergence index, & GCI $\rightarrow \mathrm{M}=3.35 \%$ GCI $_{\mathrm{M} \rightarrow \mathrm{F}}=0.95 \%$ \\
\hline GCI $(\%)$ &
\end{tabular}

\section{RESUltS AND DisCUSSION}

To understand the complex nature of the flow physics in axial flow fans, the results are presented both qualitatively and quantitatively. To validate the CFD model, firstly, the mean velocity which is measured experimentally according to log-linear rule (Winternitz and Fischl 1957) is compared with the CFD results. These mean velocities are then used to compute the volumetric flow rate by multiplying the duct area for the baseline case. The experimental and numerical results are in very good agreement as shown in Table 3 and set the way forward for the subsequent CFD simulations. Since the experiment is conducted at $1500 \mathrm{rpm}$, the CFD is also performed at the same rotational speed for the baseline case. However, the flow patterns and fan performance are computed at the maximum rotational speed of the fan, i.e., $3000 \mathrm{rpm}$. 

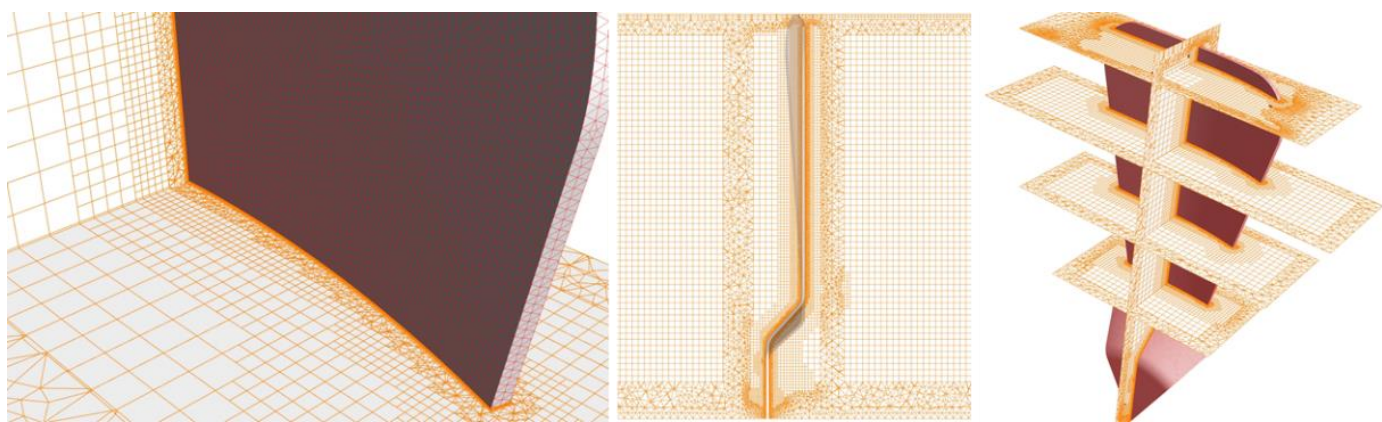

Fig. 8. Computational grid around the blade and in the fluid zones.

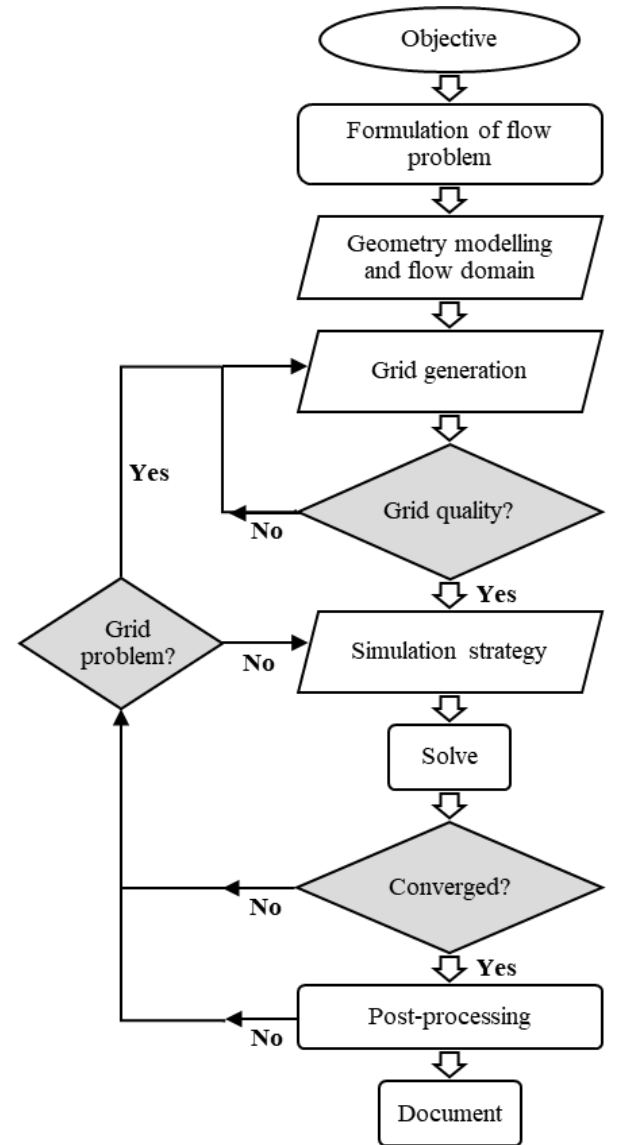

Fig. 9. The CFD process.

Table 3 Comparison of experiment and CFD results for the baseline fan configuration.

\begin{tabular}{|c|c|c|}
\hline Parameters & $\begin{array}{c}\text { Experimental } \\
\text { results }\end{array}$ & $\begin{array}{c}\text { CFD } \\
\text { results }\end{array}$ \\
\hline Flow velocity, $(\mathrm{m} / \mathrm{s})$ & 1.40 & 1.35 \\
\hline $\begin{array}{c}\text { Volumetric flow rate, } \\
Q\left(\mathrm{~m}^{3} / \mathrm{s}\right)\end{array}$ & 0.074 & 0.072 \\
\hline
\end{tabular}

\subsection{Flow Patterns}

Fig. 10 shows the static pressure distribution over the fan inlet plane. NBF has relatively lower pressure on fan inlet as compared to RBF whereas FBF show relatively more static pressure which may deteriorate the fan performance. This is evident from the average static pressure for each fan case. Figure 11 shows the static pressure contours at the fan outlet plane. The pressure is similar in NBF and RBF at the outlet plane but the FBF has very low pressure at the outlet plane which means that FBF cannot raise enough pressure to have good fan performance. Figure 12 presents the contours of static pressure at the fan blade surface. The pressure contours show a large pressure region at the tips, particularly in the NBF. However, the FBF and RBF show relatively lowpressure region on the blade compares to the NBF. The low-pressure regions at the blade surface are encircled. Figure 13 shows the static pressure distribution around the blade profile at $r / R=0.55$. NBF shows smooth pressure distribution on both suction and pressure sides. However, FBF has quite different and variable pressure at the pressure-side of the blade whereas no such variations have seen on both pressure and suction sides of RBF in the presence of beads.

\subsection{Fan Performance}

The following parameters measure the axial fan performance.

$$
\begin{aligned}
& \eta_{\text {fan }}=\frac{P_{\text {out }}}{P_{\text {in }}} \\
& P_{\text {out }}=\Delta p_{s} \cdot Q \\
& P_{\text {in }}=\Omega \cdot T \\
& \eta_{\text {fan }}=\frac{\Delta p_{s} \cdot Q}{\Omega \cdot T}
\end{aligned}
$$

Where $\eta_{\text {fan }}$ is the fan static efficiency, $P_{\text {out }}$ is the output fan power defined as the product of static pressure rise across the fan $\left(\Delta p_{s}\right)$ and volumetric flow rate $(Q)$, Pin is the input power defined as the product of fan rotational speed $(\Omega)$ and torque $(T)$. The energy efficiency $\left(E_{f}\right)$ is a key to ensure reliable and sustainable energy systems and defined as the ratio between volumetric flow rate and torque, having the units as $\mathrm{m}^{2} /$ (N.min).

$$
E_{f}=\frac{Q}{T}
$$

Fig. 14(a) shows the performances of the static pressure rise across the fan with various volume flow rates. The static pressure decreases with the increase of the fan flow rate for these three kinds of fans. The 


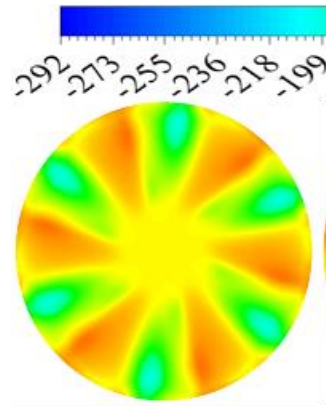

(a)

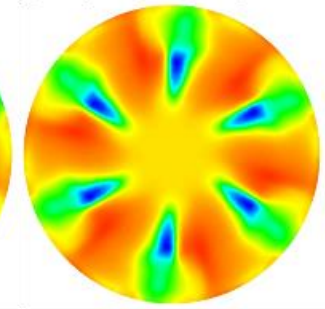

(b)

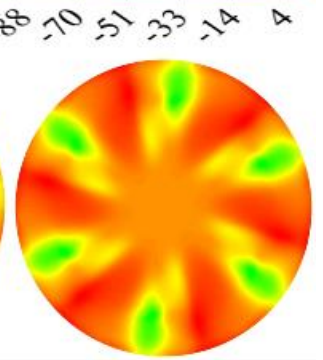

(c)

Fig. 10. Contours of static pressure at fan inlet plane at $Q=0.075 \mathrm{~m}^{3} / \mathrm{s}$ and $3000 \mathrm{rpm}$. (a) $\mathrm{NBF}$, avg. $p_{s}=$ 92Pa; (b) RBF, avg. $p_{s}=-82 \mathrm{~Pa}$; (c) FBF, Avg. $p_{s}=-47 \mathrm{~Pa}$.

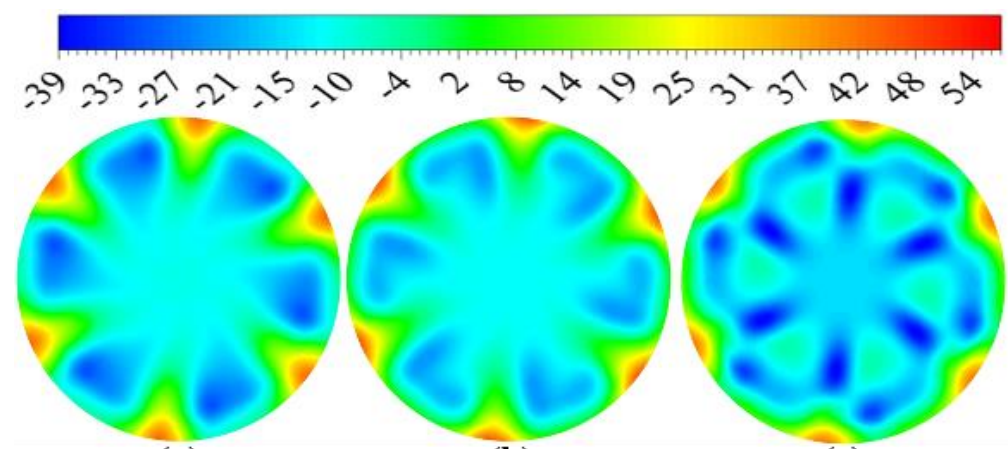

(a)

(b)

(c)

Fig. 11. Contours of static pressure at fan outlet plane at $Q=0.075 \mathrm{~m}^{3} / \mathrm{s}$ and $3000 \mathrm{rpm}$. (a) $\mathrm{NBF}$, avg. $p_{s}=$ $-6 \mathrm{~Pa}$; (b) RBF, avg. $p_{s}=-5 \mathrm{~Pa}$; (c) FBF, Avg. $p_{s}=-10 \mathrm{~Pa}$.

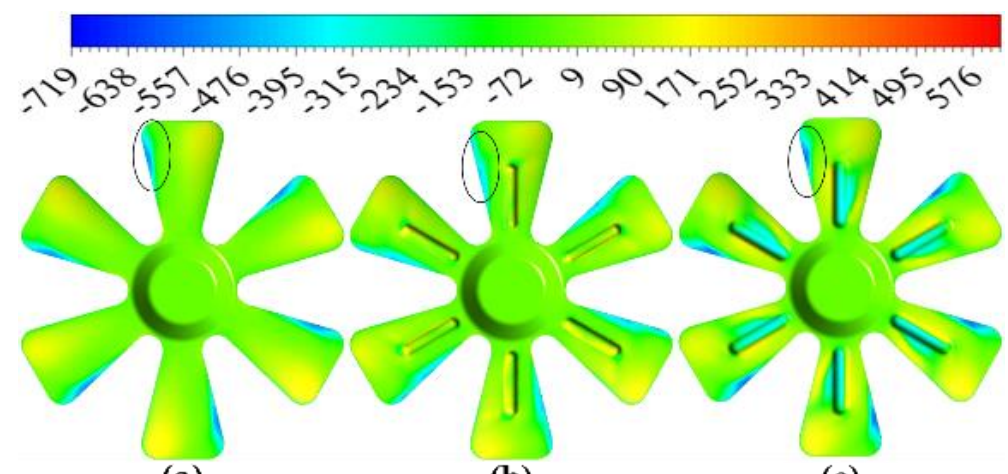

(a)

(b)

(c)

Fig. 12. Contours of static pressure at fan surface at $Q=0.075 \mathrm{~m}^{3} / \mathrm{s}$ and $3000 \mathrm{rpm}$ viewed from fan pressure side.

static pressure of NBF and RBF is the largest and comparable whereas for FBF it is the least. Fig. 14(a) also indicates the maximum volumetric flow rate which is again comparable in the case of NBF and $\mathrm{RBF}, \mathrm{FBF}$ shows the least maximum volumetric flow rate. Fig. 14(b) shows the fan static efficiency with different volumetric flow rates. The fan static efficiency increases with the increase of the flow rate achieving maximum value and starts decreasing with the increase of the fan flow rate for these three kinds of fans. The static efficiency of NBF is the largest, followed by RBF and FBF shows the least. Despite the same static pressure rise in NBF and RBF, RBF consumes more power which in results shows less efficiency than NBF. Fig. 14(c) shows the energy efficiency against different volume flow rates. The energy efficiency increases with the increase of the fan flow rate for these three kinds of fans. The energy efficiency of NBF is the largest, followed by RBF and FBF. The least energy efficiency in RBF and FBF indicates that both these fans produce more torque which in turn consumes more power. 


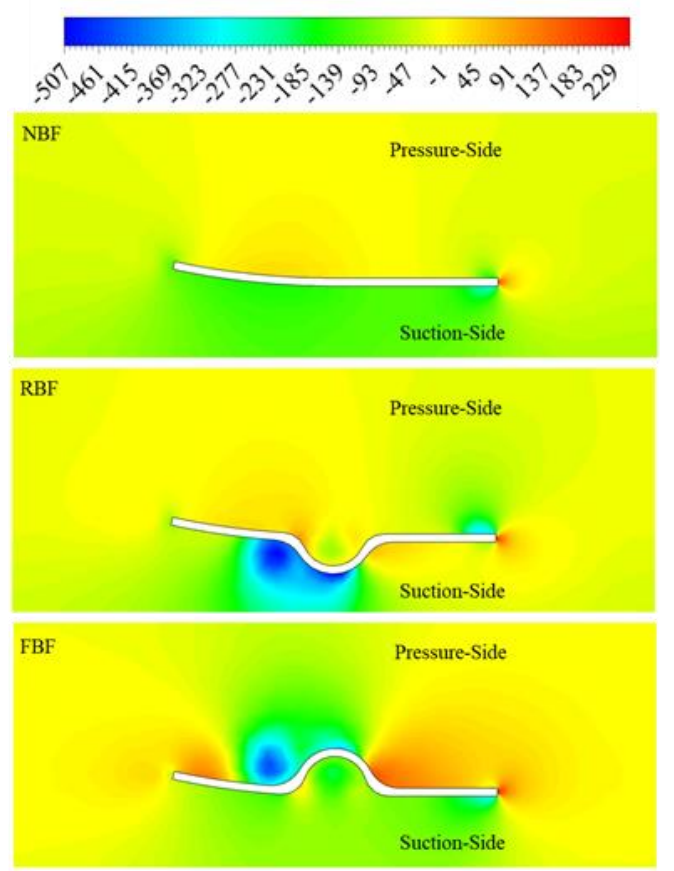

Fig. 13. Static pressure contours at around blade profile at a plane at $r / R=0.55$.

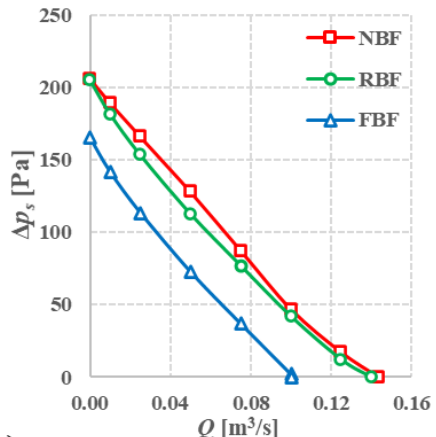

(a)

Fig. 14. Fan performance comparison of three kinds of fans (a) static pressure rise, (b) fan static efficiency, (c) fan energy efficiency as a function of volumetric flow rate.
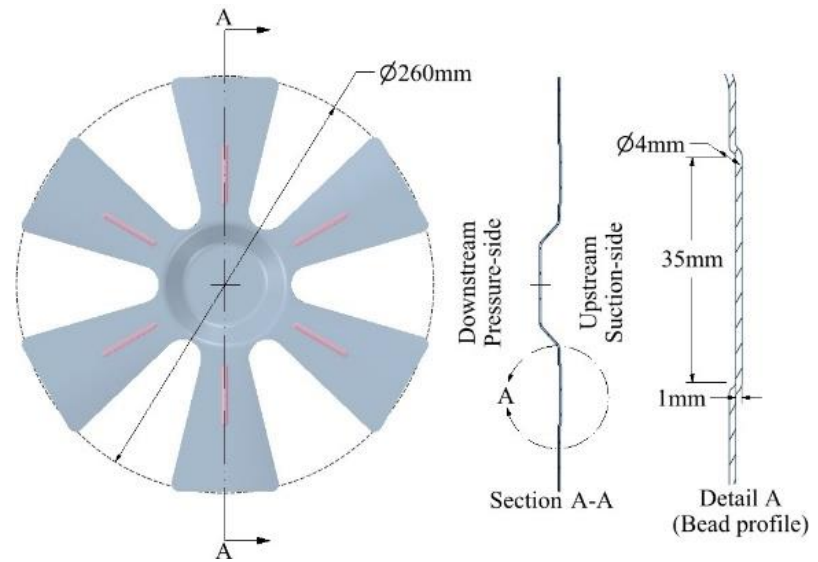

Fig. 15. Geometric illustration of the reverse-beads optimized fan (RBF-Opt).

Since the objective of the study is to increase the flow rate of the fan, Fig. 14 shows that RBF has the potential to further optimize the bead location and size to achieve more flow rate. The numerical simulations are conducted for various bead locations and sizes in RBF.

Fig. 15 shows the geometry of such a case in which 
a significant enhancement in fan performance is achieved. Fig. 16(a) shows a significant increase in static pressure rise across the RBF-Opt fan. The efficiency of RBF-Opt in Fig. 16(b) is also significantly improved than RBF but remains in the same order as NBF which presents that fans with beads consume more power than a no-beads fan. Fig. 16(c) also shows an enhancement in energy efficiency for the optimized RBF.

\subsection{Vibration Analysis}

Vibration (modal) analysis is carried out by using magnesium-alloy AZ31 material properties to evaluate the lowest mode natural frequency $\left(f_{n}\right)$ of all fan types with bead structure in comparison to the baseline fan (NBF). Table 4 shows the first six natural frequencies of fan designs at a rotational speed of $3000 \mathrm{rpm}$ specified to be the maximum rotational speed of the fan.

Table 4 Modal analysis at $3000 \mathrm{rpm}(50 \mathrm{~Hz})$.

\begin{tabular}{|c|c|c|c|c|}
\hline \multirow{2}{*}{ Mode No. } & \multicolumn{4}{|c|}{$f_{n}[\mathrm{~Hz}]$} \\
\cline { 2 - 5 } & NBF & RBF & FBF & RBF-Opt \\
\hline 1 & 77 & 79 & 79 & 76 \\
\hline 2 & 77 & 79 & 79 & 76 \\
\hline 3 & 98 & 109 & 111 & 98 \\
\hline 4 & 98 & 109 & 111 & 98 \\
\hline 5 & 104 & 121 & 121 & 105 \\
\hline 6 & 105 & 122 & 125 & 106 \\
\hline
\end{tabular}

It is of interest that lowest mode frequencies of FBF and RBF are much closer to NBF which is sufficiently far from the maximum rotational frequency of the fan (i.e., $50 \mathrm{~Hz}$ ). It seems that all fan designs are safe for vibrations.

Fig. 17 shows the natural frequency of the fan types with various rotational speeds. The natural frequency increases with the increase of the fan rotational speed for these four kinds of fans. The natural frequency coincides with RBF and FBF whereas it is also the same in NBF and RBF-Opt. At low rotational speeds, $f_{n}$ has an appreciable difference among beads and nobeads fan. However, it starts decreasing as the fan starts rotating at higher speeds.

\section{CONCLuSION}

Initially, the fan performance of three kinds of fans with and without beads is numerically investigated based on unsteady Reynolds-averaged NavierStokes equations with the sliding-mesh methodology. The baseline fan is manufactured in a 3D-printer and tested to verify the numerical results which shows a good agreement. In fans with bead structure, RBF shows a little, relatively comparable performance to NBF which is not sufficient. Further, to improve RBF performance, it is optimized by varying the locations and size of the bead on fan suction side. Significant performance enhancement is achieved with an optimized reverse-beads fan. Moreover, the modal analysis is conducted to evaluate the natural frequency modes of all fan types which is obtained satisfactory and well beyond the rotational frequency of the fan itself. The RBF-Opt produces a more volumetric flow rate and considered as the potential fan model for prototype fabrication and testing.
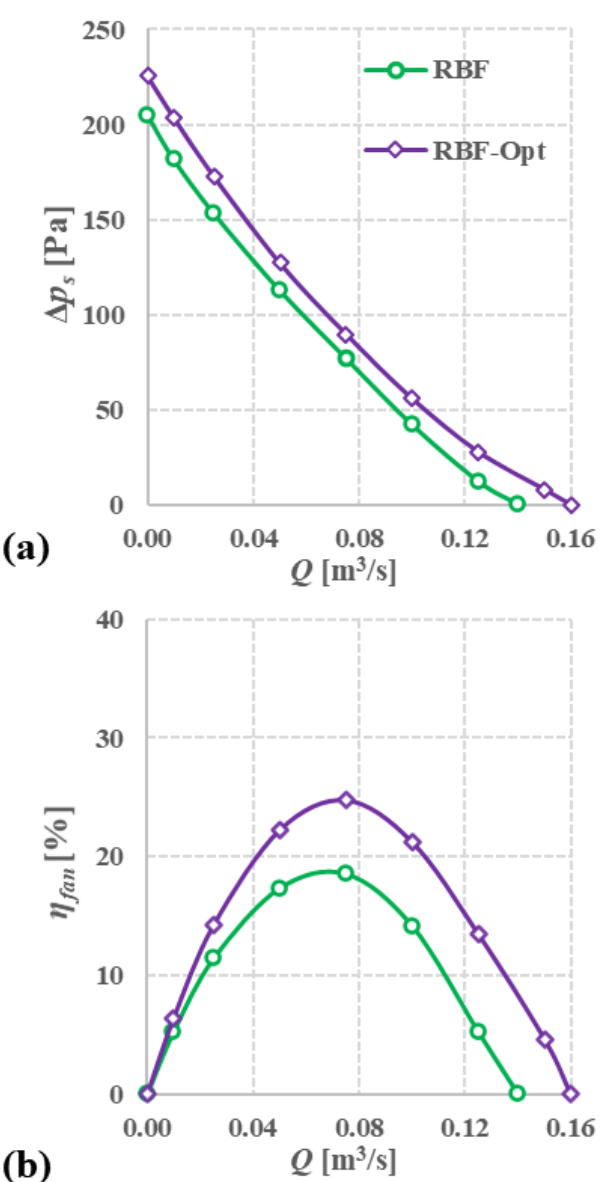

(b)

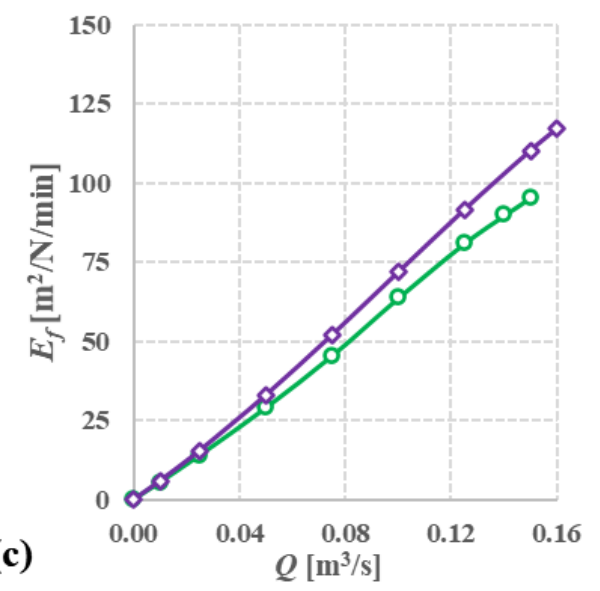

Fig. 16. Fan performance comparison of RBF and RBF-Opt (a) static pressure rise, (b) fan static efficiency, (c) fan energy efficiency as a function of volumetric flow rate. 


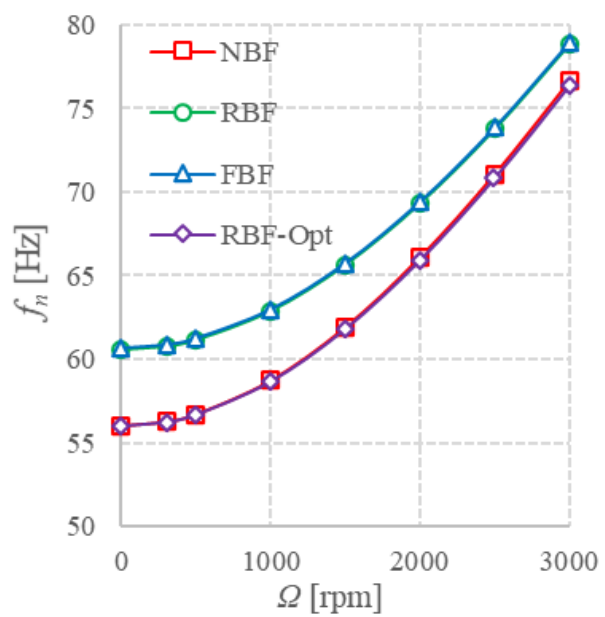

Fig. 17. The fundamental frequency of all fan types as a function of fan rotational speed.

\section{ACKNOWLEDGMENTS}

This work supported by Basic Science Research Program through the National Research Foundation (NRF) of Korea supported by Ministry of Education (Grant number: NRF-2017R1D1A1B03032472).

\section{REFERENCES}

Adeeb, E., A. Maqsood, A. Musthaq and C. H. Sohn. (2016) (2016). Parametric Study and Optimization of Ceiling Fan Blades for Improved Aerodynamic Performance. Journal of Applied Fluid Mechanics 9(6): 2905-16.

Babich, F., M. Cook, D. Loveday, R. Rawal and Y. Shukla (2017). Transient Three-Dimensional CFD Modelling of Ceiling Fans. Building and Environment 123, 37-49.

Bianchi, S., A. Corsini and A. G. Sheard (2014). A Critical Review of Passive Noise Control Techniques in Industrial Fans. Journal of Engineering for Gas Turbines and Power 136(4): 044001.

Bianchi, S., A. Corsini, A. G. Sheard and C. Tortora (2013). A Critical Review of Stall Control Techniques in Industrial Fans. ISRN Mechanical Engineering 213, 1-18.

Bleier, F. P. (1998). Fan Handbook. New York: McGraw-Hill.

Bredell, J. R., D. G. Kröger and G. D. Thiart (2006). Numerical Investigation of Fan Performance in a Forced Draft Air-Cooled Steam Applied Thermal Engineering 26(8-9): 846-52.

Chen, J., L. Li, G. Huang and X. Xiang (2018). Numerical Investigations of Ducted Fan Aerodynamic Performance with Tip-Jet. Aerospace Science and Technology 78, 51021.

Eck, B. (1973). Fans: Design and Operation of Centrifugal, Axial-Flow and Cross-Flow Fans.
Edited by R. S. Azad and D. R Scott. Oxford: Pergamon.

Gifford, N. L., A. G. Hunt, E. Savory and R. J. Martinuzzi (2006). Experimental Study of Low-Pressure Automotive Cooling Fan Aerodynamics Under Blocked Conditions. Csme, 1-8.

Gullberg, P. A. and A. B. Tavernier (2014). Modeling of Closed Fans Using CFD and Steady State Assumption of Fluid Flow. SAE Technical Papers.

Gullberg, P., L. Lofdahl and P. Nilsson (2011). Fan Modeling in CFD Using MRF Model for under Hood Purposes. In ASME-JSME-KSME 2011 Joint Fluids Engineering Conference (1), 93142.

Haider, B. A., C. H. Sohn, Y. S. Won and Y. M. Koo (2017). Aerodynamic Performance Optimization for the Rotor Design of a Hovering Agricultural Unmanned Helicopter. Journal of Mechanical Science and Technology 31 (9): 4221-26.

Haider, B. A., C. H. Sohn, Y. S. Won and Y. M. Koo. (2017). Aerodynamically Efficient Rotor Design for Hovering Agricultural Unmanned Helicopter. Journal of Applied Fluid Mechanics 10 (5): 1461-74.

Henner, M., A. Levasseur and S. Moreau (2003). Detailed CFD Modeling of Engine Cooling Fan Systems Airflow. SAE Technical Papers, no. 724 .

Hofe, R. V. and G. E. Thien (1985). Radial and Tangential Flow Fans-An Alternative to Axial Flow Fans for Low Noise Automotive Cooling Systems. SAE Technical Paper 850987.

Luo, J. Y. and A. D. Gosman (1994). Prediction of Impeller-Induced Flow in Mixing Vessels Using Multiple Frames of Reference. Institute of Chemical Engineers Symposium Series 136, 549-56.

Meyer, C. J. and D. G. Krger (2001). Numerical Simulation of the Flow Field in the Vicinity of an Axial Flow Fan. International Journal for Numerical Methods in Fluids 36 (8), 947-69.

Nobile, R., M. Vahdati, J. F. Barlow and A. Mewburn-Crook (2014). Unsteady Flow Simulation of a Vertical Axis Augmented Wind Turbine: A Two-Dimensional Study. Journal of Wind Engineering and Industrial Aerodynamics 125, 168-79.

Sahili, A., B. Zogheib and R. M. Barron (2013). 3-D Modeling of Axial Fans. Applied Mathematics 4 (4): 632-51.

Soldner, J., W. Zobel, M. Ehlers, J. Nies and J. Wattelet (2001). A Compact Cooling System (CCSTM): The Key to Meet Future Demands in Heavy Truck Cooling. SAE Technical Papers, no. 724.

Spuy, S. J. Van der, T. W. Von Backström and D. G. 
K. H. Hur et al. / JAFM, Vol. 14, No. 1, pp. 11-21, 2021.

Kröger (2010). An Evaluation of Simplified Methods to Model the Performance of Axial Flow Fan Arrays. $R \& D$ Journal of the South African Institution of Mechanical Engineering 26, 12-20.

Srinivasa, V. K., R. S. Srinivasa and B. Shome (2014). Design of Experiments Enabled CFD Approach for Optimizing Cooling Fan Performance. SAE Technical Papers 1.

Tare, K., U. Mukherjee and R. J. Vaidya (2017). Design Optimization of Automotive Radiator Cooling Module Fan of Passenger Vehicle for Effective Noise Management Using CFD Technique. SAE Technical Papers 2017-Janua (January).

Thiart, G. D. and T. W. von Backström (1993). Numerical Simulation of the Flow Field near an Axial Flow Fan Operating under Distorted Inflow Conditions. Journal of Wind Engineering and Industrial Aerodynamics 45 (2): 189-214.

Wallis, R. A. (1983). Axial Flow Fans and Ducts.
Wang, T. T., A. Jagarwal, J. R. Wagner and G. Fadel. (2015). Optimization of an Automotive Radiator Fan Array Operation to Reduce Power Consumption. IEEE/ASME Transactions on Mechatronics 20 (5): 235969.

Williams, E. E., H. Didandeh and K. M. Cahill (2011). Design of an Innovative HighEfficiency Fan for Engine Cooling. Institution of Mechanical Engineers - VTMS 10, Vehicle Thermal Management Systems Conference and Exhibition. Woodhead Publishing Limited.

Winternitz, F. A. L. and C. F. Fischl (1957). A Simplified Integration Technique for Pipe Flow Measurement. Water Power 9 (6): 22534.

Yu, J., T. Zhang and J. Qian (2011). Classification: Electric Motors, Pumps, Fans. Electrical Motor Products, 11-172. 